\title{
Weighting the inheritance coin: active genetics at work in the mouse
}

Grunwald, H.A. et al. Nature 566, 105-109 (2019)

The jerboa, says developmental biologist Kimberly Cooper, is the closest thing to a mouse that doesn't look like a mouse. Other rodents like rats and hamsters more or less share a similar murine-like body plan and proportions. The jerboa, by comparison, has (hind)legs that stretch for miles. It's a bipedal rodent that hops about on its hind legs, with three toes rather than five and metatarsals that are fused into a single bone. Cooper's lab at the University of California, San Diego wants to understand how the jerboa's distinctive limbs came to be, and what that can reveal about limb development more generally. "One of our goals is to identify genes that we think are actually responsible for evolutionary change," she says. The jerboa itself isn't a genetically tractable model at the moment, but the mouse is. If given jerboa genes, will the mouse take on its relative's more leggy attributes?

Changing individual genes in the mouse can be relatively easy now, but the Cooper lab is interested in more complicated combinations. "The evolution of the longer limb skeleton in the jerboa probably involves lots and lots of different changes that accumulated over evolutionary time," Cooper says. "We're making each of those changes [in mouse] to understand what they do individually, and we want to breed them together to try to understand what they do in combination."

That starts to get complicated-and costly, in terms of both time and animalsto pull off with current technology. To breed a triple homozygous mouse, for example, it would take 146 offspring to find just one that's likely to have inherited both mutated copies of all three genes from its parents. At least when the probability is 50/50, as Mendelian inheritance dictates; it's a random flip of a genetic coin that determines which copies, or alleles, of a gene an animal receives from its parents. Cooper and her lab decided to weight the coin.

The potential to increase the probability of inheriting a particular genetic mutation had already been demonstrated in insects (the hypothetical makings of "gene drives" that could one day aid in pest control). In a proof-of-concept paper published in Nature, Cooper and her lab bring active genetics to a mammal for the first time. They developed a construct involving CRISPR/Cas9 that
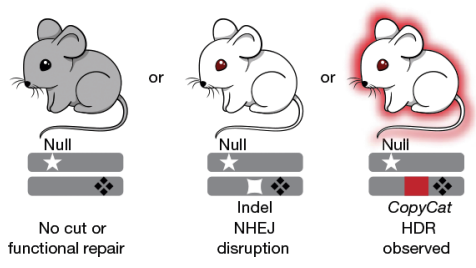

A successful outcome of Grunwald et al.'s technique produces red-glowing albino mice (far right). Reprinted with permission from Grunwald et al (2019). Springer Nature.

will break DNA at a specific place and then use an engineered genetic sequence as the template to repair the break on both alleles, a form of homology-directed repair (HDR) that should theoretically produce mouse litters that are $100 \%$ homozygous for the engineered sequence.

In their initial demonstration, the team created an active genetic element they called 'CopyCat' that they inserted into the Tyrosinase gene, which influences coat color, along with a sequence encoding red fluorescent protein. Successfully engineered, CopyCat-homozygous mice should be albino and glow red. Many glowing white mice were born. In the most efficient experiments, the researchers saw $72 \%$ inheritance of their construct-not perfect, but an improvement nonetheless from natural inheritance. "This was the first step in showing the fundamental mechanism is possible," says Cooper.

"I must say that the approach presents some intriguing possibilities for lab animal research," comments Lauryl Nutter, associate director of the Toronto Centre for Phenogenomics, which produces genetically engineered mouse models. "Such an approach is an attractive alternative to producing double or triple knockouts for research at 'super-Mendelian' ratios."

There is still variability to contend with and the current strategy is only effective in female mice-likely a complication of the timing of cell divisions in developing egg cells versus sperm relative to when Cas9 is expressed. Further work is needed.

"I do think that this manuscript, along with others exploring how to optimize HDR after Cas9-mediated DNA cleavage, highlights our need to better understand how DNA repair pathways are differentially controlled at different times during development and even at different phases of the cell cycle in mitotic cells," Nutter notes. But overall, "I think this paper is very interesting, brings to the fore some of the challenges that we would face if thinking about gene drives in a mammalian context, and will act as a springboard for efficient development of a variety of complex mouse mutants."

Whether active genetics approaches will work as efficiently with other mammalian genes remains to be seen and will likely be very project-specific, Cooper says. The alleles for the gene intended to be edited have to be different enough that the tool will only cut the 'non-desired' of the pair-the engineered version has to remain intact to serve as the repair template. Other strategies might also be need to be considered to accomplish different kinds of editing. "When thinking about using this type of technique for exploring genetic variation other than knockouts (e.g. point mutations or SNVs), one would have to envision the ability to have the guide RNA expressed from a different, though perhaps linked, locus such that it targets the non-variant allele while leaving the variant allele intact and poised to serve as an HDR template," Nutter comments. "In such a scenario, Cas9 specificity will be key."

The Cooper lab's immediate plans are to try to improve the tool's efficiency but from there, they'd like to explore whether they can weight the inheritance of multiple genes at a time. "It might never materialize because we're early in the game," Cooper acknowledges. But, "if this isn't the ideal approach to reduce the number of animals that we're using and make complicated genetics possible, maybe there are other approaches." Any improvements would be a gain, she says.

The end goal might be to make a mouse look more like a jerboa, with fewer animals required along the way, but Cooper welcomes others to see what the technique can do for their own research questions. "I'm always looking for people with interesting problems to play with," she says.

\section{Ellen Neff}

Published online: 4 March 2019

https://doi.org/10.1038/s41684-019-0261-6 